\title{
Caracterização do controle de Haematobia irritans e Rhipicephalus (Boophilus) microplus no Triângulo Mineiro e Alto Paranaíba, Minas Gerais ${ }^{1}$
}

\author{
Luísa N. Domingues ${ }^{2 *}$, Ana C.P.P. Bello², Arildo P. Cunha², Patrícia V.B. Leite ${ }^{2}$, \\ Antonio T.M. Barros ${ }^{3}$ e Romário C. Leite ${ }^{2}$
}

\begin{abstract}
Domingues L.N., Bello A.C.P.P., Cunha A.P., Leite P.V.B., Barros A.T.M. \& Leite R.C. 2012. [Characterization of Haematobia irritans and Rhipicephalus (Boophilus) microplus control in Triângulo Mineiro and Alto Paranaíba, Minas Gerais.] Caracterização do controle de Haematobia irritans e Rhipicephalus (Boophilus) microplus no Triângulo Mineiro e Alto Paranaíba, Minas Gerais. Pesquisa Veterinária Brasileira 32(12):1246-1252. Departamento de Medicina Veterinária Preventiva, Escola de Veterinária, Instituto Nacional de Ciência e Tecnologia de Informação Genético-Sanitária da Pecuária Brasileira, Universidade Federal de Minas Gerais, Av. Antônio Carlos 6627, Cx. Postal 567, Campus Pampulha, Belo Horizonte, MG 30123-970, Brazil. E-mail: dominguesln@gmail.com

The effective control of Haematobia irritans (horn fly) and Rhipicephalus (Boophilus) microplus (cattle tick) is crucial for increasing the productivity of livestock industry in Brazil, but still remains a challenge. A greater knowledge about parasite control in the field is necessary to establish control strategies closer to farmer's reality and easier to be performed. This study characterized the practices adopted for controlling such ectoparasites on 23 cattle ranches from the Triângulo Mineiro and Alto Paranaíba region in the state of Minas Gerais, through interviews. Most interviewees recognized the importance of these parasites to livestock, however, many problems related to parasite control were observed. Control of horn flies and cattle tick was a routine in $17.4 \%$ and $95.7 \%$ of the ranches, respectively, triggered by high infestations on animals. More than six insecticide/acaricide treatments were applied yearly in all cattle ranches controlling horn flies and in $76.5 \%$ of the ranches controlling the cattle tick. Pesticide products were applied mostly by manual backpack sprayers $(63.6 \%)$ without restraining the animals in $45.5 \%$ of ranches. Product dilution following manufacturer's recommendations was performed by $45.5 \%$ of interviewees, but $63.6 \%$ of them used lower doses per animal than technically recommended. Associations of organophosphates and pyrethroids were the main products used to control both parasites. Although pesticide use was a routine practice, the use of personal protective equipment (PPE) was not common among surveyed people. Most interviewees were able to recognize epidemiological characteristics of the parasites; however, parasite control measures adopted at most cattle ranches tend to jeopardize the efficacy of products, susceptibility of parasites and sustainability of control programs.
\end{abstract}

INDEX TERMS: Horn fly, Haematobia irritans, cattle tick, Rhipicephalus (Boophilus) microplus interview, parasite control.

\footnotetext{
${ }^{1}$ Recebido em 1 de maio de 2012.

Aceito para publicação em 9 de setembro de 2012.

${ }^{2}$ Departamento de Medicina Veterinária Preventiva, Escola de Veterinária, Instituto Nacional de Ciência e Tecnologia de Informação Genético-Sanitária da Pecuária Brasileira, Universidade Federal de Minas Gerais (UFMG), Av. Antônio Carlos 6627, Cx. Postal 567, Campus Pampulha, Belo Horizonte, MG 30123-970, Brasil. *Autor para correspondência: dominguesln@gmail.com

${ }^{3}$ Embrapa Pantanal, Rua 21 de Setembro 1880, Corumbá, MS 79320900, Brasil.
}

RESUMO.- 0 controle eficaz de Haematobia irritans (mosca-dos-chifres) e Rhipicephalus (Boophilus) microplus (carrapato-do-boi) é um fator crucial à maior rentabilidade da pecuária brasileira, porém ainda constitui-se um desafio. Um maior conhecimento das práticas adotadas no combate destes parasitos faz-se necessário para que se possam estruturar estratégias de controle mais próximas da realidade do produtor rural e mais fáceis de serem executadas. 
Este estudo caracterizou, através de entrevistas, as práticas adotadas no controle desses ectoparasitos em 23 propriedades da mesorregião do Triângulo Mineiro e Alto Paranaíba, Minas Gerais. A maioria dos entrevistados reconheceu a importância desses parasitos para a atividade pecuária, entretanto, diversos problemas relativos ao controle parasitário foram observados. 0 controle da mosca-dos-chifres e do carrapato-do-boi era uma prática rotineira em, respectivamente, $17,4 \%$ e $95,7 \%$ das propriedades, geralmente realizado em função de elevado grau de infestação dos animais. Mais de seis aplicações de ectoparasiticidas eram realizadas por ano em todas as propriedades que empregavam tratamentos para o controle da mosca e em $76,5 \%$ daquelas que combatiam o carrapato. Os produtos eram aplicados principalmente com bombas costais manuais $(63,6 \%)$ e sem a contenção dos animais em $45,5 \%$ das propriedades. A diluição dos produtos segundo recomendações dos fabricantes era realizada por $45,4 \%$ dos entrevistados, porém, $63,6 \%$ aplicavam um volume de solução por animal menor do que o tecnicamente recomendado. Utilizava-se principalmente a associação de piretróides e organofosforados para o controle de ambos os parasitos. Apesar de utilizar rotineiramente ectoparasiticidas, o uso de equipamento de proteção individual (EPI) não era comum entre os entrevistados. A maioria dos entrevistados conhecia algumas características epidemiológicas dos parasitos, entretanto, o controle parasitário adotado na maior parte das propriedades tende a comprometer não apenas a eficácia dos tratamentos, mas a suscetibilidade dos parasitos e a sustentabilidade do controle.

TERMOS DE INDEXAÇÃO: Mosca-dos-chifres, Haematobia irritans, carrapato-do-boi, entrevistas, Rhipicephalus (Boophilus) microplus, controle parasitário.

\section{INTRODUÇÃO}

As parasitoses dos animais determinam um significativo entrave à pecuária nacional, pois causam redução na produtividade, mortalidade de animais e aumento dos custos de produção, além de gastos com as tentativas de controle (Grisi et al. 2002, Bianchin et al. 2006). A mosca-dos-chifres, Haematobia irritans irritans (Linnaeus, 1758) (Diptera: Muscidae), e o carrapato-do-boi, Rhipicephalus (Boophilus) microplus (Canestrini, 1887) (Acari: Ixodidae), são considerados importantes ectoparasitos da pecuária bovina brasileira, causando prejuízos estimados em U\$ 865 milhões e U\$ 2 bilhões anuais, respectivamente (Grisi et al. 2002, Bianchin et al. 2006).

0 controle destes ectoparasitos baseia-se primordialmente na aplicação de ectoparasiticidas comerciais. 0 uso indiscriminado de tais produtos, juntamente com práticas inadequadas para o combate dos parasitos e a falta de especificidade dos ectoparasiticidas, tem colaborado para a rápida seleção de populações resistentes (Santos Junior et al. 2000, Saueressig \& Barros 2003, Rodrigues et al. 2004, Oliveira et al. 2006, Barros et al. 2007, Santos et al. 2009, Mendes et al. 2011).

Embora tecnicamente mais complexas, as estratégias de controle integrado de ectoparasitos são mais viáveis econômica e operacionalmente, além de serem mais próximas da realidade da maioria das propriedades pecuárias em todo o país. 0 conhecimento das características culturais e socioeconômicas das propriedades e das práticas adotadas para o controle dos parasitos é essencial para o sucesso no desenvolvimento e implantação de programas integrados de controle no campo. Sendo assim, este estudo caracterizou, através de entrevistas, o controle dos ectoparasitos $H$. irritans e R. microplus em propriedades pecuárias da mesorregião do Triângulo Mineiro e Alto Paranaíba, estado de Minas Gerais.

\section{MATERIAL E MÉTODOS}

Em abril/2010, foram realizadas entrevistas semiestruturadas em 23 propriedades localizadas na mesorregião do Triângulo Mineiro e Alto Paranaíba, MG, região com o maior rebanho e produção de leite do estado (IBGE 2009). As propriedades localizavam-se nos seguintes municípios: Água Comprida $(n=1)$, Araxá $(n=1)$, Campo Florido $(n=2)$, Conquista $(n=1)$, Ituiutaba $(n=5)$, Monte Alegre de Minas ( $n=1)$, Uberaba $(n=4)$ e Uberlândia ( $n=8)$ (Fig.1). As propriedades foram escolhidas com o apoio da Associação Brasileira dos Criadores de Girolando, sendo, portanto, uma amostragem por conveniência.

Foram entrevistados proprietários ou funcionários de propriedades onde também foram realizados bioensaios com $H$. irritans e coletadas teleóginas de $R$. microplus para avaliação da suscetibilidade destes parasitos à piretróides e organofosforados (Domingues 2011). 0 intuito das entrevistas foi conhecer e caracterizar as práticas de controle destes parasitos, uma vez que a maneira como este é realizado pode influenciar nos níveis de suscetibilidade aos ectoparasiticidas.

As entrevistas semiestruturadas - isto é, que possuíam questões fechadas e abertas, sendo estas últimas de resposta direta (Rosa \& Arnoldi 2006) - se basearam em um questionário previamente utilizado por ATM Barros e colaboradores em pesquisa realizada em diferentes estados do país, entre os anos de 2000 a 2003 (Barros et al. 2012). Os entrevistados foram questionados quanto às características das propriedades (localização, tamanho e tipo do rebanho), conhecimentos sobre a epidemiologia da mosca-dos-chifres e do carrapato-do-boi e práticas adotadas para o controle desses parasitos, tais como: momento da aplicação dos produtos ectoparasiticidas, frequência de tratamento do rebanho, produtos utilizados, modo de aplicação, local onde os animais recebiam os tratamentos, número de animais tratados, critérios de escolha e substituição dos parasiticidas e uso de equipamento de proteção individual (EPI).

Em novembro/2009 foram realizadas entrevistas-piloto em cinco propriedades da mesma mesorregião onde a pesquisa foi realizada, buscando-se adequar as questões à região e adaptar a linguagem do entrevistador para que esta fosse a mais clara e próxima possível da realidade do entrevistado (Rosa \& Arnoldi 2006).

Este foi um estudo descritivo, que não teve caráter de inquérito ou censo. A análise das entrevistas foi realizada pelo sistema de análise quantitativo-descritivo pelo qual se verificou a frequência simples de ocorrência de cada resposta, seguida pelo cálculo de porcentagem (Rosa \& Arnoldi 2006). As respostas relativas ao período de ocorrência de maiores infestações pelos ectoparasitos foram agrupadas em período chuvoso ou período seco, sendo considerados os meses de outubro a março como época chuvosa e, de abril a setembro, como época seca.

\section{RESULTADOS}

Dentre as 23 propriedades pesquisadas, $18(78,2 \%)$ possuíam um rebanho com mais de 100 animais, destacando- 


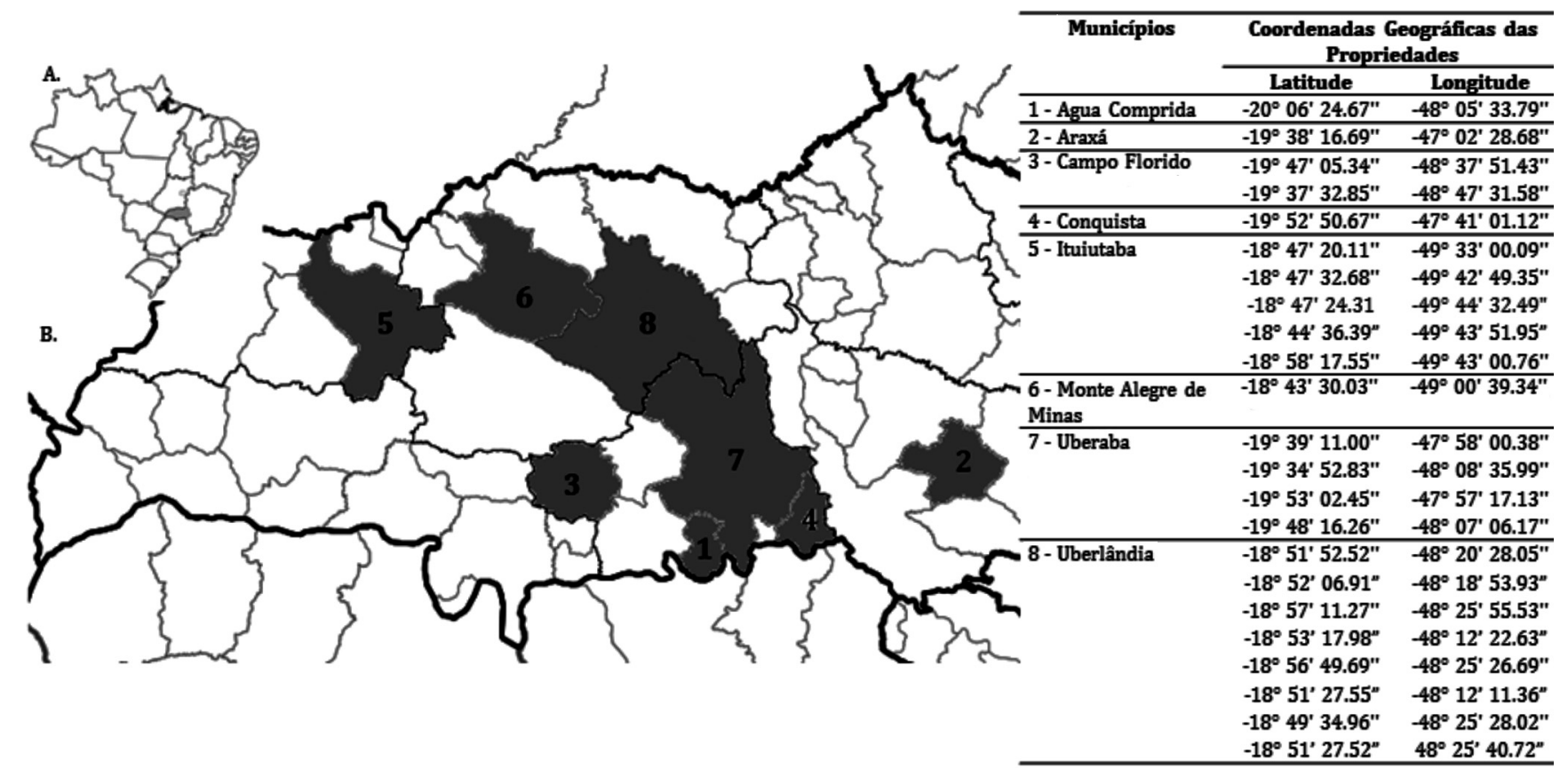

Fig.1. (A) Mapa do Brasil com destaque para a mesorregião do Triângulo Mineiro e Alto Paranaíba/ MG. (B) Municípios onde as propriedades visitadas se localizam, 2010.

-se a bovinocultura leiteira como a principal atividade pecuária desenvolvida (Quadro 1). Dentre os entrevistados, sete $(30,4 \%)$ eram proprietários e $16(69,6 \%)$ eram funcionários.

Segundo 22 (95,7\%) entrevistados, ambos os parasitos ocorriam nas propriedades, havendo diferença de infestação entre animais de diferentes graus de sangue, por ambos os parasitos. Dentre estes, $18(81,8 \%)$ afirmaram que os animais de maior grau de sangue taurino eram preferencialmente acometidos pelos parasitos, enquanto os demais não souberam especificar o motivo de tal preferência. De acordo com a maioria dos entrevistados, as maiores infestações, por ambos os parasitos, ocorriam no período chuvoso (Quadro 2).

Vinte e dois entrevistados (95,7\%) reconheceram que a mosca-dos-chifres, assim como o carrapato-do-boi, causa

Quadro 1. Tamanho e aptidão dos rebanhos de propriedades pecuárias da mesorregião do Triângulo Mineiro e Alto Paranaíba/MG, 2010

\begin{tabular}{|c|c|c|c|c|c|}
\hline \multicolumn{2}{|l|}{ Informação } & \multicolumn{3}{|c|}{ Respostas } & \multirow{2}{*}{$\begin{array}{c}\text { Total } \\
23\end{array}$} \\
\hline Número de animais & $10-50$ & $51-100$ & $101-200$ & Mais de 200 & \\
\hline & $8,7 \%$ & $13,0 \%$ & $30,4 \%$ & $47,8 \%$ & \\
\hline Aptidão & Leite & Corte & - & - & $30^{*}$ \\
\hline & $86,7 \%$ & $13,3 \%$ & & & \\
\hline
\end{tabular}

* Era permitida mais de uma resposta por entrevistado.

\section{Quadro 2. Período de maior abundância de Haematobia irritans e Rhipicephalus microplus na mesorregião do Triângulo Mineiro e Alto Paranaíba, MG,2010}

\begin{tabular}{lcc}
\hline Época/mês & Mosca-dos-chifres & Carrapato-do-boi \\
& FA (FR) & FA (FR) \\
\hline Todo o ano & $0(0,0 \%)$ & $1(4,4 \%)$ \\
Período chuvoso & $17(73,9 \%)$ & $17(73,9 \%)$ \\
Período seco & $6(26,1 \%)$ & $5(21,7 \%)$
\end{tabular}

$\overline{\text { FA }(F R) ~=~ F r e q u e ̂ n c i a ~ a b s o l u t a ~(F r e q u e ̂ n c i a ~ r e l a t i v a) . ~}$ prejuízos aos rebanhos bovinos; entretanto, apenas 17,4\% afirmaram realizar tratamentos específicos para o controle da mosca-dos-chifres. Segundo estes, os tratamentos eram realizados quando se observava uma alta carga parasitária nos animais, sendo mais de seis tratamentos durante o ano.

0 controle do carrapato-do-boi era uma prática rotineira em 22 (95,7\%) propriedades. A maioria das propriedades (17) empregava ectoparasiticidas comerciais, com variável número de tratamentos; nas demais propriedades era utilizado tratamento homeopático (2) e regulador de crescimento de insetos misturado ao sal (3) , como demonstrado no Quadro 3. Apenas quatro propriedades seguiam um calendário sanitário anual, com diferenças no esquema de aplicação dos produtos: os intervalos entre aplicações variavam de $15,21,30$ dias ou tratamentos realizados em março, maio e dezembro. A maioria dos entrevistados $(76,5 \%)$ afirmou realizar mais de seis tratamentos anuais para controlar o carrapato-do-boi (Quadro 3).

Na única propriedade onde o controle do carrapato-do-boi não era realizado, o rebanho era composto somente por fêmeas, de elevado grau de sangue zebu, receptoras de embriões. Embora este fosse um dos maiores rebanhos dentre as propriedades visitadas, não havia controle de endo e ectoparasitos.

Quanto ao modo de aplicação dos ectoparasiticidas comerciais, a aspersão $(n=11)$ e o pour-on $(n=6)$ foram os métodos citados para os tratamentos contra ambos os parasitos. A bomba costal manual foi o principal equipamento utilizado na aplicação por aspersão, geralmente realizada sem a contenção dos animais (Quadro 4).

Em relação à diluição dos produtos de aspersão, cinco $(45,4 \%)$ entrevistados afirmaram diluí-los na concentração recomendada pelo fabricante, enquanto os demais $(54,6 \%)$ utilizavam concentrações diferentes da preconizada (Quadro 4$)$. Em sete $(63,6 \%)$ propriedades, os animais eram ba- 
Quadro 3. Controle do carrapato-do-boi Rhipicephalus microplus em propriedades pecuárias da mesorregião do Triângulo Mineiro e Alto Paranaíba/MG, 2010

\begin{tabular}{|c|c|c|c|c|}
\hline Informação & & Respostas & & $\begin{array}{c}\text { Número de } \\
\text { propriedades }\end{array}$ \\
\hline Controle do carrapato & $\begin{array}{c}\text { Tratamento } \\
\text { homeopático } \\
2(9,1 \%)\end{array}$ & $\begin{array}{l}\text { Regulador de cres- } \\
\text { cimento de insetos } \\
3(13,6 \%)\end{array}$ & $\begin{array}{c}\text { Ectoparasiticidas } \\
\text { comerciais } \\
17(77,3 \%)\end{array}$ & $22(100 \%)$ \\
\hline $\begin{array}{l}\text { Momento de aplicação } \\
\text { dos ectoparasiticidas- } \\
\text { comerciais }\end{array}$ & $\begin{array}{c}\text { Grau de infestação } \\
\text { do rebanho } \\
12(70,6 \%)\end{array}$ & $\begin{array}{l}\text { Calendário Sani- } \\
\text { tário Anual } \\
4(23,5 \%)\end{array}$ & $\begin{array}{c}\text { Tamanho do } \\
\text { Carrapato } \\
1(5,9 \%)\end{array}$ & $17^{*}(100 \%)$ \\
\hline \multirow[t]{2}{*}{$\begin{array}{l}\text { Número de tratamen- } \\
\text { tos realizados por ano }\end{array}$} & 1 a 6 & Mais de 6 & $\begin{array}{l}\text { Não soube } \\
\text { responder }\end{array}$ & $17(100 \%)$ \\
\hline & $3(17,6 \%)$ & $13(76,5 \%)$ & $1(5,9 \%)$ & \\
\hline
\end{tabular}

* Somente propriedades nas quais eram utilizados ectoparasiticidas comerciais.

Quadro 4. Caracterização do uso de produtos para controle de Haematobia irritans e Rhipicephalus microplus em propriedades pecuárias da mesorregião do Triângulo Mineiro e Alto Paranaíba/MG, 2010

\begin{tabular}{|c|c|c|c|c|c|}
\hline \multirow{4}{*}{$\begin{array}{l}\text { Informação } \\
\text { Equipamento } \\
\text { de aspersão }\end{array}$} & \multicolumn{4}{|c|}{ Respostas } & \multirow{3}{*}{$\begin{array}{c}\begin{array}{c}\text { Número de } \\
\text { propriedades }\end{array} \\
11^{*}\end{array}$} \\
\hline & Bomba costal & Bomba de trator & Bomba elétrica & Câmara & \\
\hline & manual & & & atomizadora & \\
\hline & $7(63,6 \%)$ & $1(9,1 \%)$ & $1(9,1 \%)$ & $2(18,2 \%)$ & \\
\hline \multirow{2}{*}{$\begin{array}{l}\text { Forma de tratamen- } \\
\text { to por aspersão }\end{array}$} & $\begin{array}{c}\text { Animais soltos } \\
\text { no curral }\end{array}$ & Tronco & $\begin{array}{l}\text { Preso no } \\
\text { cabresto }\end{array}$ & $\begin{array}{l}\text { Câmara } \\
\text { atomizadora }\end{array}$ & $11^{*}$ \\
\hline & $5(45,5 \%)$ & $2(18,2 \%)$ & $2(18,2 \%)$ & $2(18,2 \%)$ & \\
\hline \multirow[t]{2}{*}{$\begin{array}{l}\text { Concentração } \\
\text { do produto }\end{array}$} & $\begin{array}{l}\text { Maior do que a } \\
\text { recomendada }\end{array}$ & $\begin{array}{l}\text { Recomendada } \\
\text { pelo fabricante }\end{array}$ & $\begin{array}{l}\text { Menor do que } \\
\text { a recomendada }\end{array}$ & - & $11^{*}$ \\
\hline & $2(18,2 \%)$ & $5(45,4 \%)$ & $4(36,4 \%)$ & - & \\
\hline \multirow{4}{*}{$\begin{array}{l}\text { Volume de solução/ } \\
\text { animal } \\
\text { Animais tratados/ } \\
\text { dia }\end{array}$} & Menos de $4 \mathrm{~L}$ & $\begin{array}{c}4 \text { a } 5 \mathrm{~L} \\
4(36,4 \%)\end{array}$ & Mais de $5 \mathrm{~L}$ & - & $11^{*}$ \\
\hline & Por categoria & Todos os animais & Mais infestados & - & $17^{* *}$ \\
\hline & animal & do rebanho & & & \\
\hline & $11(64,7 \%)$ & $4(23,5 \%)$ & $2(11,8 \%)$ & - & \\
\hline
\end{tabular}

* Somente propriedades que utilizavam aspersão. ${ }^{* *}$ Somente propriedades nas quais eram utilizados ectoparasiticidas comerciais.

nhados com menos de quatro litros de calda (Quadro 4). Na maioria dos casos, o tratamento era realizado por categoria (animais jovens, lactantes ou solteiros), seguido pelo tratamento de todos os animais da propriedade e, uma menor parcela, tratamento apenas dos mais infestados (Quadro 4).

A maioria dos produtos utilizados no controle da mosca-dos-chifres continha princípios ativos da classe dos piretróides $(85,7 \%)$, principalmente em associações com organofosforados $(57,1 \%)$. Embora maior diversidade de classes pesticidas fosse empregada no controle do carrapato-do-boi, os piretróides e suas associações com organofosforados representaram $50 \%$ dos produtos em uso (Quadro 5).

A alternância de produtos antiparasitários era realizada na maioria das propriedades $(78,3 \%)$, justificada pela perda da eficácia $(55,6 \%)$, preço do produto $(16,7 \%)$, disponibilidade nas lojas $(5,5 \%)$ e período de carência exigido (5,5\%). Alguns entrevistados (16,7\%) afirmaram desconhecer a finalidade da substituição do produto. 0 principal motivo atribuído à perda de eficácia foi o surgimento de resistência no carrapato ou na mosca $(n=8)$, seguido de problemas na fabricação dos produtos $(\mathrm{n}=1)$. A maioria dos entrevistados $(95,7 \%)$ afirmou haver assistência veterinária na propriedade. Entretanto, os veterinários eram consultados somente em casos de emergência em $52,7 \%$ das propriedades, sendo fonte de informação sobre controle parasitário em apenas sete (31,8\%) (Fig.2).

Somente em sete propriedades $(31,8 \%)$ os equipamentos de proteção individual (EPI) eram utilizados, sendo que em apenas três $(13,63 \%)$, o equipamento completo (roupa, boné, luvas, máscaras e botas) era empregado. Em várias propriedades havia o equipamento de proteção, porém o trabalhador preferia não utilizá-lo.

Quadro 5. Composição dos produtos utilizados no controle da mosca-dos-chifres e do carrapato-do-boi em propriedades pecuárias da mesorregião do Triângulo Mineiro e Alto Paranaíba/MG, 2010

\begin{tabular}{lcc}
\hline \multicolumn{1}{c}{ Classe dos produtos } & $\begin{array}{c}\text { Mosca-dos-chi- } \\
\text { fres* FA (FR) }\end{array}$ & $\begin{array}{c}\text { Carrapato-do- } \\
\text { boi* FA (FR) }\end{array}$ \\
\hline Piretróides & $2(28,6 \%)$ & $14(21,9 \%)$ \\
Piretróides + Organofosforados & $4(57,1 \%)$ & $18(28,1 \%)$ \\
Organofosforados + Organofosforados & - & $6(9,4 \%)$ \\
Avermectinas & - & $6(9,4 \%)$ \\
Reguladores de crescimento de insetos & $1(14,3 \%)$ & $8(12,5 \%)$ \\
Formamidina & - & $4(6,2 \%)$ \\
Fenilpirazol & - & $3(4,7 \%)$ \\
Diflubenzuron & - & $3(4,7 \%)$ \\
Homeopatia & - & $2(3,1)$
\end{tabular}

$\overline{\text { FA (FR) = Frequência Absoluta (Frequência Relativa). }}{ }^{*}$ Era permitida mais de uma resposta por entrevistado. 


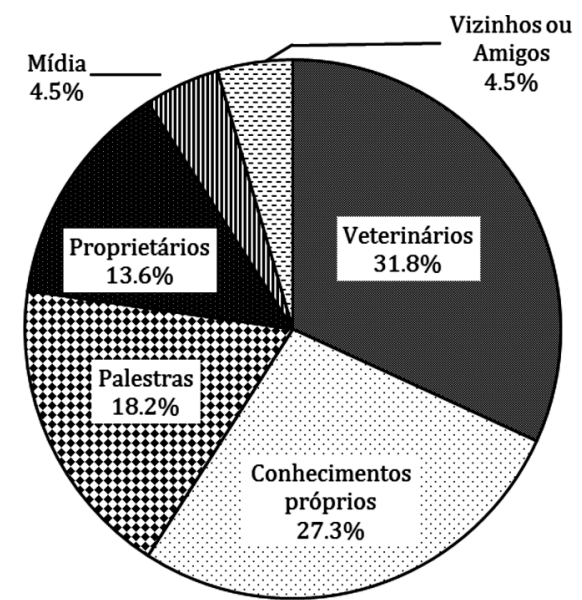

Fig.2. Fonte de informação sobre os métodos de controle dos parasitos em propriedades pecuárias da mesorregião do Triângulo Mineiro e Alto Paranaíba/MG, 2010.

\section{DISCUSSÃO}

De modo geral, pode-se afirmar que a maioria dos entrevistados no presente estudo, tanto produtores quanto funcionários, possuía conhecimento sobre aspectos epidemiológicos da mosca-dos-chifres e do carrapato-do-boi, porém realizava o controle sem critérios técnicos. Os relatos dos entrevistados sobre os períodos de maiores infestações por ambos os parasitos (Quadro 2) estão de acordo com estudos sobre a dinâmica populacional destas espécies em diferentes estados do país (Oliveira \& Freitas 1997, Lima et al. 2000, Barros 2001), bem como o reconhecimento da predileção da mosca-dos-chifres e do carrapato-do-boi por animais taurinos (Lima et al. 2000, Veríssimo et al. 2002, Bianchin et al. 2006).

Apesar dos produtores relatarem a presença e a importância de Haematobia irritans nos rebanhos, seu controle não consistia em prática rotineira, sendo decorrente das ações de combate ao carrapato. Aqueles entrevistados que realizavam um controle específico da mosca-dos-chifres, o faziam em função do aumento das infestações no rebanho, totalizando mais de seis ao ano. As recomendações para o controle da mosca-dos-chifres nas regiões Sudeste e Centro-Oeste do Brasil incluem dois tratamentos estratégicos ao ano, em todo o rebanho, sendo um no início e outro no final da estação chuvosa (Barros 1992, Leite et al. 2010). Portanto, as práticas realizadas nas propriedades estudadas na mesorregião do Triângulo Mineiro e Alto Paranaíba não estão de acordo com o preconizado para o controle da mosca-dos-chifres no País. Além disso, a elevada frequência dos tratamentos realizados aumenta a pressão de seleção e contribui para acelerar o desenvolvimento de resistência nas populações deste parasito (Georghiou \& Taylor 1977).

O controle do carrapato-do-boi mediante a observação de grande quantidade das formas adultas sobre os bovinos e a realização de mais de seis tratamentos durante o ano, conforme observado no presente estudo (Quadro 3), são práticas comuns em diversos estados brasileiros (Santos Júnior et al. 2000, Rocha et al. 2006, 2011b, Farias et al. 2008, Mendes et al. 2011).
De acordo com diversos autores (Furlong et al. 2003, Pereira et al. 2008, Leite et al. 2010, Cunha 2011), o controle do carrapato-do-boi deve ser realizado considerando-se seus aspectos bioecológicos e de dinâmica populacional, de modo a concentrar as ações de combate em somente um período do ano e reduzir o número de tratamentos anuais, os gastos com mão-de-obra, a quantidade de produtos carrapaticidas, além de prevenir resíduos dos antiparasitários nos produtos de origem animal. Para Furlong et al. (2003) e Pereira et al. (2008), na região Sudeste do Brasil, o controle do carrapato-do-boi deve ser iniciado no período quente e chuvoso do ano (primavera), com intervalos de 21 dias ou de acordo com a duração do ciclo parasitário e o período residual do produto carrapaticida, devendo perdurar por, no mínimo, quatro meses. De modo distinto, Leite et al. (2010) e Cunha (2011) preconizam, para a mesma região, que os tratamentos sejam iniciados em abril e mantidos por 120 dias, com aplicações dos produtos em todo o rebanho sempre que se observar fêmea semi-ingurgitada de tamanho igual ou maior a $3 \mathrm{~mm}$ em pelo menos um animal do rebanho.

Percebe-se, portanto, que a prática adotada para o controle de Rhipicephalus microplus nas propriedades visitadas neste estudo não está de acordo com as recomendações técnicas disponíveis e ainda contribui para o desenvolvimento de resistência nas populações do parasito. Como demonstrado por Jonsson et al. (2000) e Bianchi et al. (2003), a realização de mais de seis tratamentos durante o ano é um fator de risco para o desenvolvimento de resistência à piretróides no carrapato-do-boi.

É interessante ressaltar que mesmo em propriedades que utilizavam diariamente produto homeopático ou regulador de crescimento de insetos, fazia-se uso do controle químico tradicional quando a infestação alcançava níveis considerados inaceitáveis pelo produtor. Esta situação evidencia que tais produtos, da forma como utilizados nessas propriedades, não mantinham as infestações em níveis baixos durante todo o ano. Situação semelhante foi encontrada por Bello (2010), no estado do Rio de Janeiro, na qual a manutenção do uso dos produtos homeopáticos pelos produtores foi atribuída ao acompanhamento mensal feito pelos representantes da empresa fornecedora e à praticidade de seu uso, que exigia apenas que o produto (em pó) fosse espalhado no cocho.

Nas regiões Sudeste, Centro-Oeste e Nordeste do Brasil predomina o uso de aspersão com bombas costais, bem como a aplicação de um volume menor de inseticida/acaricida que o recomendado para o controle desses parasitos (Saueressig \& Barros 2003, Rodrigues et al. 2004, Rocha et al. 2006, 2011b, Oliveira et al. 2006, Barros et al. 2007, Mendes et al. 2011). 0 mesmo método e problema de aplicação foram encontrados no presente estudo (Quadro 4) e, em última análise, contribuem para comprometer ainda mais a eficácia dos produtos utilizados. De acordo com Pereira et al. (2008), o uso de quatro a cinco litros de calda inseticida/carrapaticida por animal adulto (cerca de um litro por $100 \mathrm{~kg}$ de peso vivo), bem como a contenção dos animais, são práticas obrigatórias para obter-se uma adequada eficácia da aplicação por aspersão. 
No presente estudo, apenas $23,5 \%$ dos entrevistados (Quadro 4) afirmaram tratar todos os animais do rebanho em um mesmo dia. Rocha et al. (2006, 2011b) encontraram situação divergente nas propriedades de Passos e Divinópolis, MG, quando aproximadamente $80 \%$ dos entrevistados tratavam todos os animais no mesmo dia. A falta de infraestrutura adequada para aplicação dos produtos em larga escala, comum nas propriedades visitadas, pode ter contribuído para a realidade encontrada no Triângulo Mineiro e Alto Paranaíba.

De modo geral, os produtos inseticidas utilizados para o controle de H. irritans na região estudada (Quadro 5) coincidem com o observado em outros estados, onde predominam os piretróides e suas associações com os organofosforados (Saueressig \& Barros 2003, Rodrigues et al. 2004, Oliveira et al. 2006, Barros et al. 2007). Segundo dados do Compêndio de Produtos Veterinários (2011), existem 95 produtos registrados para o controle da mosca-dos-chifres no Brasil, sendo 29 compostos por piretróides e 25 por associações entre piretróides e organofosforados. Portanto, a realidade encontrada nas propriedades reflete o mercado nacional de produtos inseticidas e demonstra a dificuldade que o produtor tem de mudar de classe inseticida, uma vez que as opções são restritas.

Em relação aos produtos empregados para o controle do carrapato-do-boi (Quadro 5), Mendes et al. (2011) também encontraram maior frequência de utilização de piretróides e associação de piretróides com organofosforados no estado de São Paulo. No Rio Grande do Sul, os piretróides eram mais utilizados entre 1997 e 2000, quando, então, foram gradativamente substituídos pelas formamidinas (2000 a 2006), devido ao desenvolvimento de resistência a piretróides nas populações de R. microplus em decorrência de seu uso com maior frequência e em menores concentrações, para o controle da mosca-dos-chifres (Farias et al. 2008).

A principal justificativa citada pelos entrevistados para a alternância de produtos foi a perda de eficácia, mesma realidade encontrada por Rodrigues et al. (2004) e Barros et al. (2007) em relação ao controle de H. irritans no Mato Grosso do Sul e por Rocha et al. (2006) em relação ao controle de R. microplus no estado de Minas Gerais. É interessante observar que nenhum entrevistado citou o uso inadequado como causa para a perda de eficácia dos produtos. Este fato demonstra a falta de percepção de produtores e trabalhadores rurais locais de que a grande frequência de tratamentos por ano, a falta de infraestrutura adequada e falhas relacionadas ao preparo e aplicação dos produtos sejam causas primárias de redução de eficácia e problemas de controle. Observou-se, na maioria das propriedades visitadas, uma grande preocupação com a produtividade animal, entretanto, o manejo sanitário do rebanho não recebia semelhante atenção.

Diante da pequena participação dos médicos veterinários como fonte de informação sobre os produtos e métodos de controle disponíveis para combate dos ectoparasitos (Fig.2), ressalta-se a necessidade de uma maior participação destes profissionais, bem como de instituições de pesquisa, cooperativas e associações ligadas ao setor, na divulgação dos programas de controle existentes e na capacitação dos trabalhadores rurais, para que se alcance sucesso na implantação de programas sanitários, conforme também observado por Santos Júnior et al. (2000), Bello (2010) e Rocha et al. (2006, 2011a,b).

A falta de uso de EPI pelos trabalhadores rurais, observada no presente estudo, demonstra a pouca preocupação com a saúde laboral destes trabalhadores, o que foi igualmente observado por Rocha et al. (2006, 2011b), em Minas Gerais, e Bello (2010), no Rio de Janeiro. Segundo Bello (2010), os trabalhadores justificavam o não uso do EPI por calor, falta de hábito ou falta de praticidade na rotina de trabalho, queixas também apresentadas pelos entrevistados no presente estudo. Bello (2010) ressaltou a necessidade de um programa de educação continuada dos trabalhadores rurais, visando sua conscientização sobre a importância do uso de equipamentos de proteção, com esforços concretos para redução dos problemas de saúde nas áreas rurais.

Devido ao alto custo e às dificuldades técnicas associadas ao desenvolvimento e registro de novas classes pesticidas, é fundamental a implantação de estratégias mais eficazes de controle de parasitos, que utilizem os antiparasitários de maneira criteriosa, retardando o desenvolvimento de resistência e prevenindo a presença de resíduos tóxicos no ambiente e em alimentos de origem animal.

Agradecimentos.- À Fundação de Amparo à Pesquisa do Estado de Minas Gerais (Fapemig) (Proc. APQ 0056908) e ao Conselho Nacional de Desenvolvimento Científico e Tecnológico (CNPq) (Proc. 140038/2008-6) pelo apoio financeiro concedido para a realização deste estudo. À Ricardo Canesso Dalla Rosa (Departamento de Medicina Veterinária Preventiva, Escola de Veterinária, UFMG) pelo suporte técnico prestado durante o desenvolvimento deste estudo.

\section{REFERÊNCIAS}

Barros A.T.M., Saueressig T.M., Gomes A., Koller W.W., Furlong J., Girão E.S., Pinheiro A.C., Alves-Branco F.P.J., Sapper, M.F.M, Braga R.M. \& Oliveira, A.A. 2012. Susceptibility of the horn fly, Haematobia irritans irritans (Diptera: Muscidae), to insecticides in Brazil. Revta Bras. Parasitol. Vet. 21(2):125-132.

Barros A.T.M., Gomes A. \& Koller W.W. 2007. Insecticide susceptibility of horn flies, Haematobia irritans (Diptera: Muscidae), in the state of Mato Grosso do Sul, Brazil. Revta Bras. Parasitol. Vet. 16(3):145-151.

Barros A.T.M. 1992. Recomendações para o controle da mosca-dos-chifres no Pantanal. Comun. Téc. 10, Embrapa Pantanal, Corumbá. 4p.

Barros A.T.M. 2001. Dynamics of Haematobia irritans irritans (Diptera: Muscidae) infestation on Nelore cattle in the Pantanal, Brazil. Mem. Inst. Oswaldo Cruz 96(4):445-450.

Bello A.C.P.P. 2010. A representação social do saber de trabalhadores rurais sobre o controle das parasitoses em propriedades produtoras de leite. Tese de Doutorado em Ciência Animal, Escola de Veterinária, Universidade Federal de Minas Gerais, Belo Horizonte, MG. 66p.

Bianchi M.W., Barré N. \& Messad S. 2003. Factors related to cattle infestation level and resistance to acaricides in Boophilus microplus tick populations in New Caledonia. Vet. Parasitol. 25(11):1-15.

Bianchin I., Koller W. \& Detmann E. 2006. Sazonalidade de Haematobia irritans no Brasil Central. Pesq. Vet. Bras. 26(2):79-86.

Compêndio de Produtos Veterinários 2009. SINDAN. Disponível em <http://www.sindan.org.br/sd/sindan/index.html> Acesso em 1 abr. 2011.

Cunha A.P. 2011. Controle integrado de parasitos em bovinos de leite. Tese de Doutorado em Ciência Animal, Escola de Veterinária, Universidade Federal de Minas Gerais, Belo Horizonte. 86p. 
Domingues L.N. 2011. Práticas de controle e perfil de suscetibilidade de Haematobia irritans irritans (Linnaeus, 1758) (Diptera: Muscidae) e Rhipicephalus (Boophilus) microplus (Canestrini, 1887) (Acari: Ixodidae) à cipermetrina e clorpirifós na mesorregião do Triângulo Mineiro e Alto Paranaíba, MG, 2010. Tese de Doutorado em Ciência Animal, Escola de Veterinária, Universidade Federal de Minas Gerais, Belo Horizonte. $62 \mathrm{p}$.

Farias N.M., Ruas J.L. \& Santos T.R.B. 2008. Análise da eficácia de acaricidas sobre o carrapato Boophilus microplus, durante a última década, na região do Rio Grande do Sul. Ciência Rural 38(6):1700-1704.

Furlong J., Martins J.R.S. \& Prata M.C.A. 2003. Carrapato-dos-bovinos: controle estratégico nas diferentes regiões brasileiras. Comum. Téc. 36, Embrapa Gado de Leite, Juiz de Fora. 6p.

Georghiou G.P. \& Taylor C.E. 1977. Genetic and biological influences in the evolution of insecticide resistance. J. Econ. Entomol. 70(3):319-323.

Grisi L., Massard C.L., Moya Borja G.E. \& Pereira J.B. 2002. Impacto econômico das principais ectoparasitoses em bovinos no Brasil. Hora Vet. 21(125):8-10.

IBGE 2009. Pesquisa da Pecuária Municipal. Instituto Brasileiro de Geografia e Estatística. Disponível em <http://www.sidra.ibge.gov.br/bda/ pesquisas/ppm/default.asp> Acesso em 1 abr. 2011.

Jonsson N.N., Mayer D.G. \& Green P.E. 2000. Possible risk factors on Queensland dairy farms for acaricide resistance in cattle tick (Boophilus microplus). Vet. Parasitol. 88(1):79-92.

Leite R.C., Cunha A.P., Bello A.C.P.P., Domingues L.N. \& Bastianetto E. 2010. Controle dos ectoparasitos em bovinocultura de corte, p.1171-1196. In: Pires A.V. (Ed.), Bovinocultura de Corte. Vol.2. FEALQ Piracicaba.

Lima W.S., Ribeiro M.F. \& Guimarães M.P. 2000. Seasonal variation of Boophilus microplus (Canestrini, 1887) (Acari: Ixodidae) in cattle in Minas Gerais state, Brazil. Trop. Anim. Health Product. 32(6):375-380.

Mendes M.C., Lima C.K.P., Nogueira A.H.C., Yoshihara E., Chiebao D.P., Gabriel F.H., Ueno T.E., Namindome A. \& Klafke G.M. 2011. Resistance to cypermethrin, deltamethrin and chlorpyriphos in populations of Rhipicephalus (Boophilus) microplus (Acari: Ixodidae) from small farms of the state of São Paulo, Brazil. Vet. Parasitol. 178(4):383-388.

Oliveira G.P. \& Freitas A.R. 1997. Comportamento da Haematobia irritans em fazendas com diferentes manejos de bovinos. Ciência Rural 27(2):279-284.

Oliveira A.A.A., Azevedo H.C., Melo C.B. \& Barros A.T.M. 2006. Susceptibilidade da mosca-dos-chifres (Haematobia irritans) a inseticidas nos tabu- leiros costeiros de Alagoas, Bahia e Sergipe, Brasil. Revta Bras. Parasitol. Vet. 15(2):65-70.

Pereira M.C., Labruna M.B., Szabó M.P.J. \& Klafke G.M. 2008. Rhipicephalus (Boophilus) microplus: Biologia, controle e resistência. MedVet, São Paulo. 169p.

Rocha C.M.B.M., Oliveira P.R., Leite R.C., Cardoso D.L., Calic S.B. \& Furlong J. 2006. Percepção dos produtores de leite do município de Passos, MG, sobre o carrapato Boophilus microplus (Acari: Ixodidae), 2001. Ciência Rural 36(4):1235-1242.

Rocha C.M.B.M., Leite R.C., Bruhn F.R.P., Guimarães A.M. \& Furlong J. 2011a. Perceptions about the biology of Rhipicephalus (Boophilus) microplus among milk producers in Divinópolis, Minas Gerais. Revta Bras. Parasitol. Vet. 20(4):289-294.

Rocha C.M.B.M., Leite R.C., Bruhn F.R.P., Guimarães A.M. \& Furlong J. 2011b. Perceptions of milk producers from Divinópolis, Minas Gerais, regarding Rhipicephalus (Boophilus) microplus control. Revta Bras. Parasitol. Vet. 20(4):295-302.

Rodrigues S. R., Sanches C.S., Fialho E.M.L.M., Ismael A.P.K. \& Barros A.T.M. 2004. Comercialização e uso de produtos inseticidas para controle da mosca-dos-chifres em Aquidauana, MS. . Bolm Pesq. Desenvolv. 32, Embrapa Pantanal, Corumbá. 23p.

Rosa M.V.F.P.C. \& Arnoldi M.A.G.C. 2006. A Entrevista na Pesquisa Qualitativa: mecanismos de validação dos resultados. Autêntica, Belo Horizonte. $112 p$.

Santos T.R.B., Farias N.A.R., Cunha Filho N.A., Pappen F.G. \& Vaz Junior I.S. 2009. Abordagem sobre o controle do carrapato Rhipicephalus (Boophilus) microplus no sul do Rio Grande do Sul. Pesq. Vet. Bras. 28(1):6570.

Santos Júnior J.C.B., Furlong J. \& Daemon E. 2000. Controle do carrapato Boophilus microplus (Acari: Ixodidae) em sistemas de produção de leite da Microrregião Fisiográfica Fluminense do Grande Rio, Rio de Janeiro. Ciência Rural 30(2):305-311.

Saueressig T.M. \& Barros A.T.M. 2003. Diagnóstico da susceptibilidade de populações de mosca-dos-chifres a inseticidas em Goiás, Tocantins e Distrito Federal. Bolm Pesq. Desenvolv. 82. Embrapa Cerrados, Planaltina, DF. $16 \mathrm{p}$.

Veríssimo C.J., Otsuk I.P., Deodato A.P., Lara M.A.C. \& Bechara G.H. 2002. Infestação por carrapatos Boophilus microplus (Acari: Ixodidae) em vacas Gir, Holandesa e mestiça sob pastejo. Arqs Inst. Biológico, São Paulo, 69:87-89. 